\title{
Original IMPACT OF INDUCTION CHEMOTHERAPY WITH WEEKLY PACLITAXELAND Article CISPLATIN FOLLOWED BY CHEMORADIATION ON THE MANAGEMENT OF ADVANCED HEAD AND NECK CANCER
}

\author{
Ezzat Safwat ${ }^{1}$, Ahmed Hafez ${ }^{2}$
}

${ }^{1}$ Clinical Oncology Department (NEMROCK), Faculty of Medicine, Cairo University, ${ }^{2}$ Surgical Department, ElSalam Oncology Center, Ministry of Health, Cairo, Egypt

\begin{abstract}
Background: Paclitaxel and Cisplatin are among the most active antitumor agents in head and neck cancer, and phase I studies found the combination of the two drugs to be feasible. The EORTC ECSG performed a multicenter phase II study in patients with locally advanced, recurrent or metastatic squamous cell carcinoma of the head and neck to evaluate the antitumor efficacy and toxicity of this combination.

Aim of the Study: to investigate the anti-tumor activity and toxicity profile of the combination of paclitaxel and cisplatin as induction (neoadjuvant) chemotherapy for patients with locally advanced inoperable squamous cell carcinoma of the head and neck, followed by concomitant chemoradiotherapy using cisplatin as radiosensetizer. This trial also evaluates the response rate, disease free and overall survival in this subset of patients.

Patients and Methods: Thirty eligible patients with head and neck cancer had been subjected to combination of paclitaxel (weekly) and cisplatin (every three weeks) as induction in the form of The chemotherapy regimen employed in the present study consisted of Paclitaxel 80mg/m2 administered on days 0, 7, 14, 21, 28 and 35. Cisplatin $75 \mathrm{mg} / \mathrm{m} 2$ was administered on days 0 and 21 after paclitaxel followed 2 weeks later by concomitant chemoradiotherapy using cisplatin as radiosensetizer in the form of Radiotherapy on day 49 (standard 3 shrinking field technique (6 MV photons) and weekly cisplatin at a dose of $25 \mathrm{mg} / \mathrm{m} 2$. Objective response according to WHO criteria was evaluated twice in this study following induction chemotherapy (initial response) and following chemoradiation (final response).

Results: the thirty eligible patients were subdivided into nasal related tumors (NPC) (11 patients) and non-nasal related tumours (non-NPC) (19 patients). Tumours were T3 in $19(55.9 \%)$ and T4 in $15(44.1 \%)$, N0 was in $6(17.6 \%) \mathrm{N} 1-2$ in $23(68.7 \%)$ and $\mathrm{N} 3$ in $5(14.7 \%)$. Initial responders were $24(70.5 \%)$ and increased to $29(96.7 \%)$ in final response. Concerning initial response, NPC group showed statistically higher response compared to non-NPC more in T3 than in T4. Multivariate analysis for the initial response revealed that the most deterministic factors were primarly site of the tumor, followed by the performance status, age and nodal status. Concerning overall survival and disease free, median could not be achieved in the follow up period (24 months) while at 24 months, the survival was $83 \%$ for all patients, $91 \%$ for NPC and 79\% for non-NPC. The disease free progression was $77 \%, 81 \%$ and $74 \%$ respectively. Acute toxicities were mainly hematological and gastrointestinal in all cases (100\%). Dermatological toxicities were mainly alopecia (100\%), and sensory neuropathy (40\%).

Conclusion: Induction chemotherapy with weekly paclitaxel and three-weekly cisplatin followed by concomitant chemoradiation proved its efficacy in short and intermediate term follow up in patients with unresectable head and neck tumours. Primary site, nodal status, performance status together with age are very deterministic for response of induction protocol.
\end{abstract}

Key Words: Head and neck cancer, chemoradiation, paclitaxel.

Corresponding Author: Ezzat Safwat Saad, Kasr El Aini Centre of Radiation Oncology (NEMROCK),

Tel.: +20122188452 E-mail: Ezzat_onc@msn.com

\section{INTRODUCTION}

The majority of patients with head and neck cancer present with locally advanced disease. While early stage disease is potentially curable with standard treatments of surgery and radiation, long term disease-free and overall survival rates for patients with advanced disease are poor. Approximately $50-60 \%$ of patients have local disease recurrence within 2 years, and $20-30 \%$ of patients develop metastatic disease. ${ }^{1,2}$

In an effort to improve outcomes, chemotherapy has been integrated into a combined modality approach including surgery and/or radiation therapy for locally advanced head and neck cancer. Effective strategies have incorporated chemotherapy as neoadjuvant (induction) therapy, delivered prior to definitive locoregional treatment, or concurrently with radiation therapy (Chemoradiotherapy). Data from randomized trials have confirmed that the addition of chemotherapy to curative treatment improves clinical outcomes in patients with advanced disease, demonstrating significant benefits in 
terms of organ preservation ${ }^{3-5}$, longer time to disease progression $^{3-11}$, better locoregional control ${ }^{11}$, fewer distant metastases ${ }^{5,6}$, and longer overall survival times ${ }^{6-12}$. Induction chemotherapy with cisplatin and 5-flurouracil has become a standard regimen for patients with locally advanced head and neck cancer, producing overall response rates of $60-90 \%$, with complete responses in up to $50 \%$ of patients. ${ }^{12,13}$

Although the combination of cisplatin and ${ }^{5}$ flurouracil is considered standard therapy, newer data suggest that the combination of cisplatin and paclitaxel may have equal efficacy with less toxicity ${ }^{3}$. Response data for the use of single-agent paclitaxel in patients with head and neck cancer are also well established ${ }^{4}$. In vitro studies combining paclitaxel with cisplatin demonstrated a synergistic interaction between these ${ }^{2}$ agents, whereby paclitaxel inhibited platinum-DNA adduct repair. Studies also indicate that sequencing of these agents, with cisplatin given after paclitaxel, is crucial for such synergy. ${ }^{5}$

In addition to this synergistic effect, toxicity from the combination of cisplatin and paclitaxel also has influenced the sequencing of these two agents. In phase I trials, more pronounced neutropenia was observed when cisplatin was given prior to paclitaxel. Pharmacologic data indicate that the increased toxicity probably is caused by a $25 \%$ decrease in paclitaxel clearance when cisplatin administration precedes that of paclitaxel. Other toxicities included mild-to-moderate neurotoxicity, which was more prominent in patients with pre-existing neuropathy ${ }^{6}$. The aim of this trial was to investigate the anti-tumor activity and toxicity profile of the combination of paclitaxel (weekly) and cisplatin (every three weeks) administered as induction (neoadjuvant chemotherapy for patients with locally advanced inoperable squamous cell carcinoma of the head and neck, followed by concomitant chemoradiotherapy using cisplatin as radiosensetizer. This trial also evaluates the response rate, disease free and overall survival in this category of patients.

\section{PATIENTS AND METHODS}

This trial was conducted at ElSalam Oncology Center, Minstry of Heslth during the period from September 2002 till August 2004.

\section{Eligibility:}

Newly diagnosed patients with pathologically proven, unresectable, locoregional squamous cell carcinoma of the head and neck (SCCHN), were eligible:

- $\quad$ Age $>18$ years, with measurable disease as defined by using the Response Evaluation Criteria in Solid Tumours (RECIST) ${ }^{8}$.

- $\quad$ No prior surgery, chemotherapy or radiotherapy.
- $\quad$ ECOG performance status 0-2.

- $\quad$ All patients had to have adequate renal functions as documented by a serum creatinine level $<1.5 \mathrm{mg} / \mathrm{dL}$ or a creatinine clearance $>50$ cc per minute.

- In addition, every patient had to have an absolute neutrophil count (ANC)>1500 uL, a platelet count $>100,000 \mathrm{uL}$, a serum bilirubin level $<2$ times the upper limit of normal (ULN), and alanine and aspartate aminotransferase level $<1.5$ times the ULN.

- Tumours were considered unresectable when surgical evaluation estimated resection not technically feasible or surgical radicality not acheivable despite of a significant loss of organ and/or organ function.

Pretreatment evaluation included medical history, physical and ENT examination, computerized Tomography scan (CT) of the tumour site and the neck lymph nodes, chest X-rays, endoscopy of the upper aerodigestive tract (if indicated), a complete blood count and biochemistry. Disease was staged according to the 1997 UICC TNM staging system. ${ }^{13}$

All patients were subjected to dental prophylaxis advice and had their height and body weight checked.

Patients with sensory neuropathy greater than grade two were not eligible. Patients who had uncontrolled hypertension, unstable angina, congestive heart failure, or recent myocardial infarctions within the prior 6 months were considered ineligible along with patients who had another malignancy within 5 years of enrollment.

\section{Treatment plan:}

The chemotherapy regimen employed in the present study consisted of Paclitaxel $80 \mathrm{mg} / \mathrm{m}^{2}$ administered on days $0,7,14,21,28$ and 35 . Cisplatin $75 \mathrm{mg} / \mathrm{m}^{2}$ was administered on days 0 and 21 after paclitaxel.

Cisplatin was administered during forced hydration with 2 litres normal saline solution containing potassium chloride $20 \mathrm{mEq}$ and magnesium sulphate $2 \mathrm{~g}$. Paclitaxel was given in $500 \mathrm{ml}$ of normal saline as a 3hour infusion before cisplatin. Standard premedication and antiemetic regimen were given before the administration of chemotherapy.

Two weeks rest were allowed after the end of this chemotherapy regimen during which initial assessment of response was performed.

Radiotherapy started on day 49 and weekly cisplatin at a dose of $25 \mathrm{mg} / \mathrm{m}^{2}$ was administered concomitant with the radiation. Radiotherapy employed the standard 
3 shrinking field technique (6 MV photons) with shielding block designed to protect critical organs. After a cumulative dose of 40 Gy the brain stem and spinal cord were excluded from irradiation and high energy (8$10 \mathrm{MeV}$ ) electron beams were used to treat the posterior regions of the neck. Treatment was given to all cases in $2 \mathrm{~Gy}$ daily fractions to the ICRU reference point five times weekly up to a total planned dose of 66 Gy to the clinically involved volumes. A dose of 50 Gy was delivered to clinically uninvolved electively treated lymph nodes.

\section{Treatment evaluations and adverse events:}

The National Cancer Institute Common Toxicity Criteria (version 2.0) were used for the classification of adverse events. ${ }^{9}$

Hospitalization of patients developing grade > three stomatitis or grade 4 haematological toxicity was mandatory in order to permit a more adequate adherence to the prescribed treatment protocol. GranulocyteColony Stimulating Factor (G-CSF) was administered to patients with ANC $<750$ prior to paclitaxel single agent or $<1200$ prior to paclitaxel and cisplatin combination chemotherapy. All patients with grade 3-4 stomatitis were offered either oral or parental nutritional support.

\section{Response criteria:}

Objective response according to $\mathrm{WHO}$ criteria ${ }^{14}$ was evaluated twice in this study. The initial response to neoadjuvant chemotherapy was evaluated after the initial phase of chemotherapy and before starting radiotherapy; between week 6 and 8 of the prescribed treatment protocol. The final analysis of response was performed 6 weeks after the completion of treatment using the same methods of initial staging.

Patients achieving complete remission, stationary disease or progression after neoadjuvant chemotherapy proceeded to concomitant chemoradiohterapy; while patients showing partial response after induction chemotherapy were surgically examined to assess for radical resection and if proved feasible, were given the option of continuing in the study versus proceeding to radical surgical resection. Early death was defined as any death occurring before the end of treatment.

\section{Statistical analysis:}

Major endpoints of the study were locoregional control, disease free survival , two year overall survival, response and toxicity. The statistical analysis of patients survival and disease free survival were based on comparison of Kaplan-Mayer curves by the log rank test ${ }^{15}$. Survival was estimated from the date of first treatment day to death or last follow-up visit. Disease free survival was estimated from the date of first treatment day to first evidence of disease progression. Comparison between number and percentages were done by test of proportion. $\mathrm{P}$ value $<0.05$ was considered significant.

\section{RESULTS}

Between September 2002 and August 2004, 34 patients with locally advanced squamous cell carcinoma of the upper aerodigestive tract presented to ElSalam Oncology Center and were enrolled. Two early deaths (non-treatment related cause) occurred during the induction chemotherapy and two patients underwent radical surgery following the neoadjuvant chemotherapy both of them showed partial response, hence the total number of evaluable patients who received the prescribed treatment protocol in this study is 30 patients.

\section{Patients characteristics:}

As shown in table (1), age of patients ranged from 18 to 75 years old with median of 54 years. There were 25 males $(73.5 \%)$ and 9 females $(26.5 \%)$. Performance status was 0 in $4(11.8 \%), 1$ in $23(67.6 \%)$, and 2 in $7(20.6 \%)$. Smoking habit was found in 26 patients $(76.5 \%)$.

Primary carcinoma was found in larynx in 14 (41.2\%), nasopharynx in $9(36.8 \%)$, tounge $3(6.7 \%)$, nasal cavity 2 (5.7\%), post cricoid 2 (5.7\%), cheek 2 (5.7\%), Lip 1 $(2.9 \%)$, and floor of mouth $1(2.9 \%)$. Patients with T3 were 19 (55.9\%), and T4 15 (44.1\%), while lymh node was free (N0) in $6(17.6 \%)$, involved as N1-2 in 23 $(68.7 \%)$, and $\mathrm{N} 3$ in $5(14.7 \%)$.

For further assessment of the study patients were subdivided into two main subgroups nasal tumours (nasopharynx and nasal cavity) 11 cases and non nasal tumours including 23 cases. These were distributed according to tumour size $(\mathrm{T})$ and nodal status $(\mathrm{N})$ as seen in table (2).

\section{Response assessment:}

Response post-neoadjuvant chemotherapy (initial response):

Table (1) showed the initial response (following neoadjuvant chemotherapy) was $24 / 34(70.6 \%)$ in the form of complete response $6 / 34(17.6 \%)$, and partial response 18 (52.9\%). Two of those partial responders were subjected to surgical salvage. Rest of patients were non responders in the form of stationary disease (6, $17.6 \%$ ) and progressive disease (2, 5.7\%). Early death were recorded in two cases before evaluation of the response and excluded from the study. These were case no.(2) with cancer larynx who suffered from progressive disease and died from stridor inspite of emergency tracheostomy. 
Table 1:Patients Characteristics (34 patients).

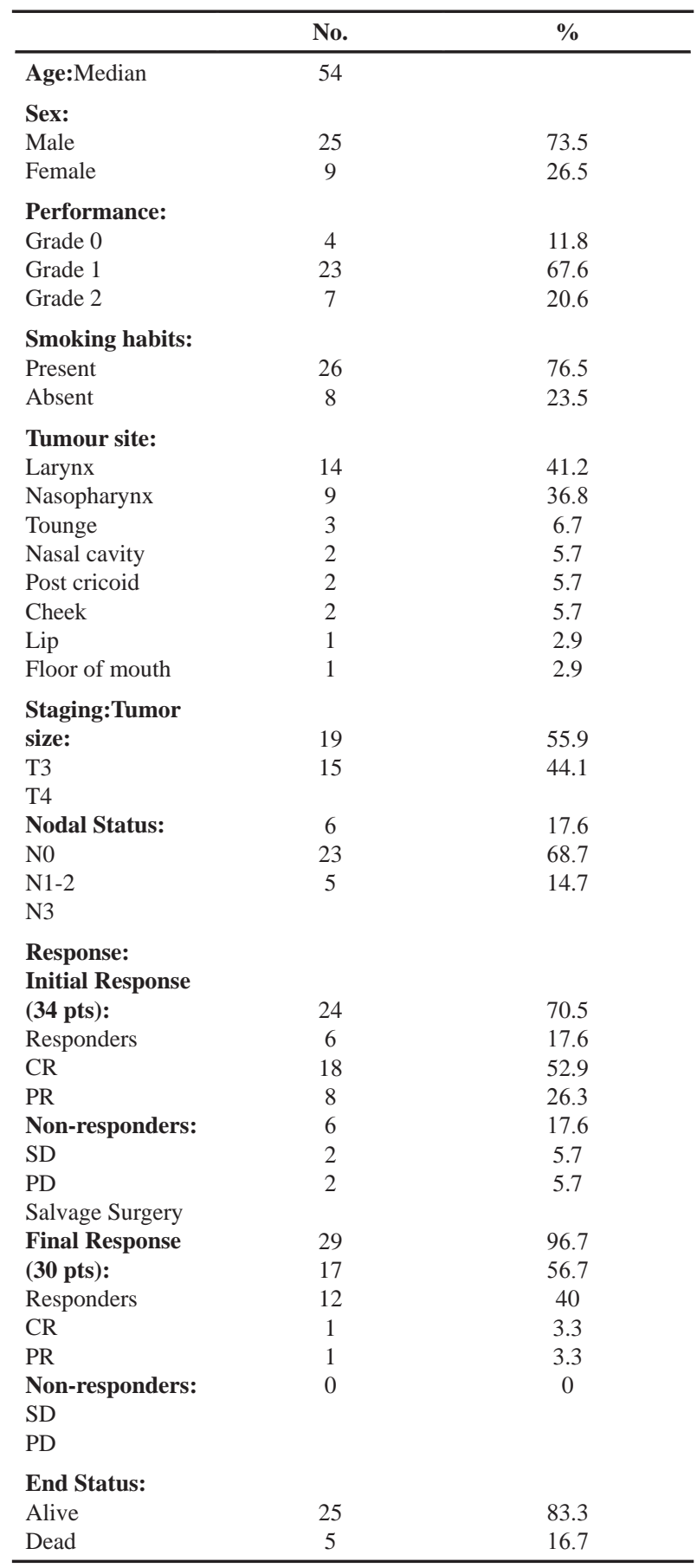

And case no. (23) with cancer tongue who suffered from progression of the disease. Two cases, one with laryngeal cancer and the other with carcinoma of the cheek(buccal mucosa), both achieved partial response to induction chemotherapy and were given the surgical option. Both were subjected to radical surgery and excluded on the assessment of response to radiotherapy.

Table (3) showed impact of site of the primary tumour whether nasal of non-nasal and tumour size and nodal status on the response to neoadjuvant chemotherapy. It was found that patients with nasal tumours (nasopharynx and nasal cavity) showed complete response $(6 / 6,100 \%)$ in case of T3 versus 8/12 (66.7\%) in non-nasal tumours. However, incidence of response was significantly reduced in NPC group in case of T4 to be 4/5 (80\%) but yet higher than in non-NPC (66.7\%).

On doing multivariate analysis including regression analysis to detect the most important factors affecting response to chemotherapy in the studied patients, it had been found that tumour primary site is the most important, followed by performance status, and age. The later when introduced made lymph nodal status is more important than tumour size (Table 4). This was followed by analysis of these deterministic factors on complete response and non-responders as seen in table (5).

\section{Response post-radiotherapy (final response):}

After exclusion of the four cases, rest of patients legible for the current study were 30 patients. Accordingly, table (1) showed that responders increase in number 29/30 (96.7\%). On the other hand, figure (1) revealed impact of radiotherapy on different patients pattern of response in such way that out of the 18 cases with initial partial response 11 showed complete response (61.1\%). On the other hand, $7 / 8$ patients $(87.5 \%)$ of non responders showed partial response post radiotherapy.

Table 2:Distribution of studied patients according to tumour size (T) and nodal status (N).

\begin{tabular}{|c|c|c|c|c|c|c|c|c|c|c|c|c|c|c|}
\hline & \multicolumn{6}{|c|}{ T3 } & \multicolumn{6}{|c|}{ T4 } & \multicolumn{2}{|c|}{ Total } \\
\hline & \multicolumn{2}{|c|}{ NPC } & \multicolumn{2}{|c|}{ Non-NPC } & \multicolumn{2}{|c|}{ Total } & \multicolumn{2}{|c|}{ NPC } & \multicolumn{2}{|c|}{ Non-NPC } & \multicolumn{2}{|c|}{ Total } & & \\
\hline & No. & $\%$ & No. & $\%$ & No. & $\%$ & No. & $\%$ & No. & $\%$ & No. & $\%$ & No. & $\%$ \\
\hline No & 0 & 0 & 1 & 7.7 & 1 & 5.3 & 2 & 40 & 3 & 30 & 5 & 33.3 & 6 & 17.6 \\
\hline N1 & 3 & 50 & 1 & 7.7 & 4 & 21.0 & 1 & 40 & 4 & 40 & 5 & 33.3 & 9 & 26.5 \\
\hline N2 & 3 & 50 & 6 & 46.1 & 9 & 47.4 & 2 & 40 & 3 & 30 & 5 & 33.3 & 14 & 41.2 \\
\hline N3 & 0 & 0 & 5 & 38.5 & 5 & 26.3 & 0 & 0 & 0 & 0 & 0 & 0 & 5 & 14.7 \\
\hline Total & 6 & 17.6 & 13 & 38.2 & 19 & 55.9 & 5 & 14.7 & 10 & 29.4 & 15 & 44.1 & 34 & 100 \\
\hline
\end{tabular}

$\mathrm{NPC}=$ Nasal carcinoma, non-NPC $=$ Non-nasal carcinoma 
Table 3:Impact of tumor size $(\mathrm{T})$ and nodal status $(\mathrm{N})$ on response to neoadjuvant chemotherapy.

\begin{tabular}{|c|c|c|c|c|c|c|c|c|c|c|c|c|}
\hline & \multicolumn{6}{|c|}{ T3 } & \multicolumn{6}{|c|}{$\mathrm{T} 4$} \\
\hline & \multicolumn{3}{|c|}{ NPC } & \multicolumn{3}{|c|}{ Non-NPC } & \multicolumn{3}{|c|}{ NPC } & \multicolumn{3}{|c|}{ Non-NPC } \\
\hline & CR & PR & NR & CR & PR & NR & CR & PR & NR & CR & PR & NR \\
\hline N0 & 0 & 0 & 0 & 0 & 1 & 0 & 0 & 2 & 0 & 1 & 1 & 1 \\
\hline N1-2 & 4 & 2 & 0 & 0 & 6 & 1 & 0 & 2 & 1 & 1 & 3 & 2 \\
\hline N3 & 0 & 0 & 0 & 0 & 1 & 3 & 0 & 0 & 0 & 0 & 0 & 0 \\
\hline \multirow[t]{2}{*}{ Total } & 4 & 2 & 0 & 0 & 8 & 4 & 0 & 4 & 1 & 2 & 4 & 3 \\
\hline & \multicolumn{2}{|c|}{$6 / 6 * *(100 \%)$} & $<0.01^{*}$ & \multicolumn{3}{|c|}{$8 / 12 * * *(66.7 \%)$} & \multicolumn{2}{|c|}{$4 / 5 * *(80 \%)$} & $<0.05^{*}$ & \multicolumn{3}{|c|}{$6 / 9 * * *(66.7 \%)$} \\
\hline
\end{tabular}

*P between NPC and non-NPC responders ** $\mathrm{P}<0.05$ between T3 and T4 in NPC group $* * * \mathrm{P}>0.05$ between T3 and T4 of non-NPC group

Table 4: Multivariate analysis of factors affecting response to neoadjuvant chemotherapy.

\begin{tabular}{lcc}
\hline & R & P \\
\hline Tumor site (NPC as 2 vs non-NPC as 1) & 0.73 & $<0.00001$ \\
Performance status & -0.52 & $<0.001$ \\
Age & -0.37 & $<0.01$ \\
Lymph node status & -0.36 & $<0.01$ \\
\hline
\end{tabular}

$\mathrm{r}=$ correlation coefficient

Table 5: Simple Analysis of determinstic Factors derived from the multivariate analysis in table 4, on complete response and non responders.

\begin{tabular}{|c|c|c|c|c|c|}
\hline Case No & Site of tumour & Performance Status (PS) & $\mathbf{T}$ & $\mathbf{N}$ & Age \\
\hline \multicolumn{6}{|c|}{ Complete Responders } \\
\hline 6 & NPX & 0 & 3 & 2 & 18 \\
\hline 12 & NPX & 1 & 3 & 2 & 47 \\
\hline 19 & NPX & 1 & 3 & 1 & 28 \\
\hline 25 & Larynx & 0 & 4 & 0 & 50 \\
\hline 33 & Tounge & 1 & 4 & 1 & 57 \\
\hline 34 & NPX & 1 & 3 & 1 & 62 \\
\hline Simple analysis & $\mathrm{NPX}=4 / 6(66.7 \%)$ & All cases $<2$ & T3 4/6 (66.7\%) & N0-1 4/6 (66.7\%) & $43.7+5.8$ \\
\hline \multicolumn{6}{|c|}{ Non-Responders } \\
\hline 7 & Larynx & 1 & 3 & 3 & 41 \\
\hline 11 & Larynx & 1 & 4 & 1 & 37 \\
\hline 13 & Larynx & 2 & 4 & 0 & 75 \\
\hline 14 & Postcricoid & 1 & 4 & 1 & 57 \\
\hline 22 & Larynx & 1 & 3 & 2 & 56 \\
\hline 26 & Larynx & 1 & 3 & 3 & 44 \\
\hline 28 & NPX & 1 & 4 & 2 & 27 \\
\hline 30 & Tounge & 2 & 3 & 3 & 69 \\
\hline Simple analysis & Larynx 5/8 (62.5\%) & PS 2 in 2/8 (25\%) & T4 in $4 / 8(50 \%)$ & $\mathrm{N} 2-3$ in $5 / 8(62.5 \%)$ & $51+8.9$ \\
\hline
\end{tabular}

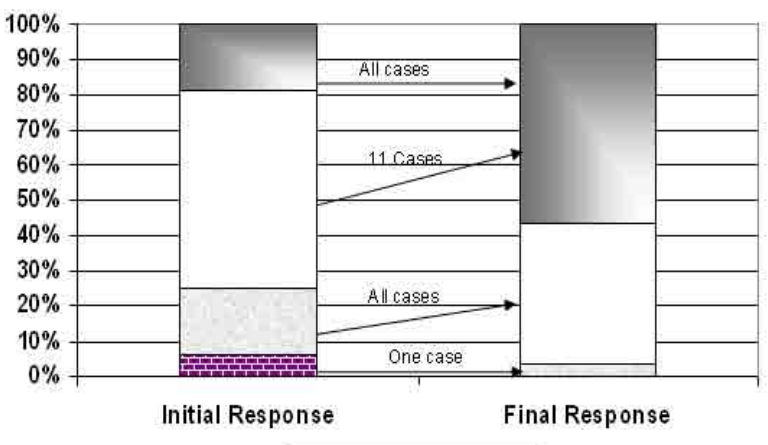

国DP $\square \mathrm{SD} \square \mathrm{PR} \square \mathrm{CR}$

Fig.1: Distribution of the Studied Patients according to the Initial and Final response 


\section{Concerning toxicity:}

Table (6) revealed different type of toxicity for combination of both chemotherapy and radiotherapy in such way that.

\section{Survival:}

Figures (2) and (3) revealed total overall survival and time to disease progression where median of survival

Table 6: Acute Toxicities (30 patients).

\begin{tabular}{|c|c|c|c|c|c|c|}
\hline & \multicolumn{2}{|c|}{ Total number affected } & \multicolumn{2}{|c|}{ Grade 1-2 } & \multicolumn{2}{|c|}{ Grade 3-4 } \\
\hline & No. & Percentage of total (30) & No. & Percentage of total (30) & No. & Percentage of total (30) \\
\hline \multicolumn{7}{|c|}{ Hematological } \\
\hline Leucopenia & 30 & 100 & 18 & 60 & 12 & 40 \\
\hline Anaemia & 30 & 100 & 23 & 76.7 & 7 & 23.3 \\
\hline Thrombocytopenia & 13 & 43.3 & 11 & 36.7 & 2 & 6.7 \\
\hline \multicolumn{7}{|c|}{ Gastrointestinal } \\
\hline Stomatitis & 20 & 100 & 14 & 46.7 & 16 & 53.3 \\
\hline Nausea \& Vomiting & 23 & 76.6 & 15 & 50 & 8 & 26.7 \\
\hline \multicolumn{7}{|c|}{ Dermatological \& neurological } \\
\hline Alopecia & 30 & 100 & 17 & 56.7 & 13 & 43.3 \\
\hline $\begin{array}{l}\text { Sensory } \\
\text { Neuropathy }\end{array}$ & 12 & 40 & 11 & 36.7 & 1 & 3.3 \\
\hline $\begin{array}{l}\text { Hypersenstivity } \\
\text { reaction }\end{array}$ & 3 & 10 & 3 & 10 & 0 & 0 \\
\hline \multicolumn{7}{|c|}{ Renal Function } \\
\hline Impairment & 3 & 10 & 3 & 10 & 0 & 0 \\
\hline
\end{tabular}

Hematological toxicity: Leucopenia was found in the 30 patients $(100 \%)$ but $18 / 30(60 \%)$ grade $1-2$ toxicity, and 12 (40\%0 with grade 3-4. Anemia was found in the 30 patients with $23(76.7 \%)$ grade $1-2$ and $7(23.3 \%)$ grade $3-4$. Thrombocytopenia in 13 (43.3\%) with grade $1-2$ in $11 / 13(84.6 \%)$ grade $1-2$ and $2 / 13(15.4 \%)$ grade 3-4.

GIT toxicity: Stomatitis was recorded in all patients with $14 / 30(46.7 \%)$ grade $1-2$ and $16 / 30(54.3 \%)$ grade $3-4$. Nausea and vomiting was recorded in 23/30 (76.7\%) in the form of grade 1-2 in 15/23 (65.2\%) and grade 3-4 in $8 / 23(34.8 \%)$.

Dermatological toxicity: Alopecia was found in all patients with grade $1-2$ in $17 / 30(56.7 \%)$ and grade $3-4$ in 13/30 (43.3\%). Hypersensitivity was recorded in on 3 cases (10\%) as grade 1-2.

Sensory neuropathy was recorded in 12/30 (40\%) as grade 1 -2 in $11 / 12(91.7 \%)$ and grade $3-4$ in $1 / 12$ (8.3\%).

Nineteen patients (43\%) required parenteral nutrition due to severe mucositis, and two patients needed red blood cell transfusions

Renal toxicity: renal functional impairment grade 1-2 was recorded in 3/30 (10\%) of cases.

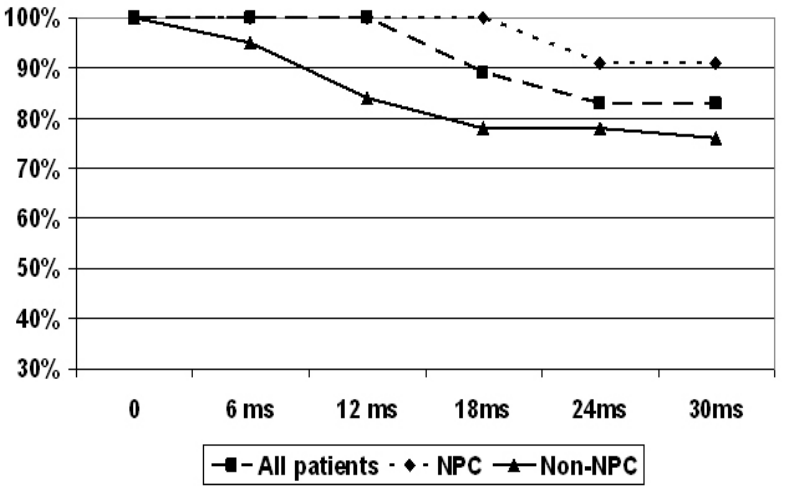

Fig. 2: Total Actuarial Of The Studied Groups

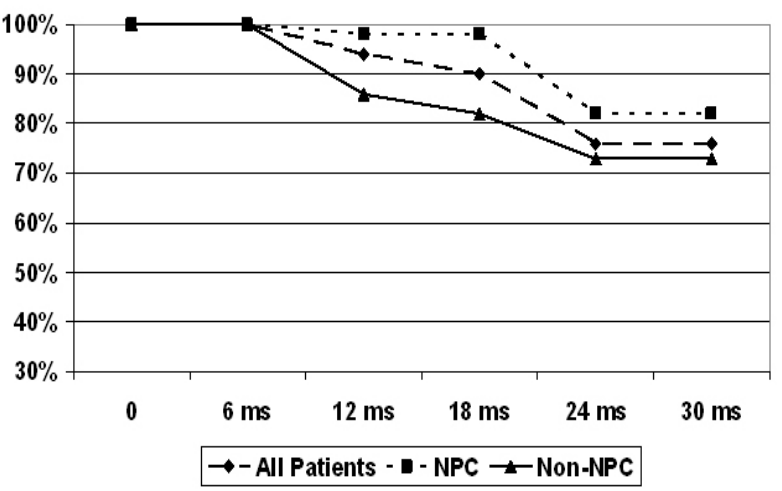

Fig. 3: Time To Disease Progression In The Studied Groups. 
could not be recorded in the current study during the follow up period of 24 months.

\section{Outcome:}

In February 2006, with a follow-up time of 24 months (range 18-30 months), 23 of 30 patients( $76.6 \%$ ) had not progressed, and 7 patients (23.4\%) had shown disease progression (locoregional and distant metastasis). These patients had been subjected to palliative chemotherapy and showed partial response in three cases, stationary disease in two cases and disease progression in two cases. The estimated 2-year time to disease progression (TTP) was $77 \%$ (Figure 3 ). The sites of disease progression were the primary tumor sites in two cases, neck lymph nodes in two cases, distant metastases to the lungs and bones in three cases. Five of the relapsing patients died and two were still alive with disease at the end of study. The total actuarial survival was found to be not reached in all patients or in both groups separately, however, at 2 years-follow up the overall survival was found to be $83 \%$, 91\%, and 79\% for all patients, NPC and non NPC, respectively.

\section{DISCUSSION}

New anticancer agents, including taxanes, and innovative drug combinations for treatment of HNC are currently being evaluated in clinical trials. Paclitaxel achieved objective response rates of $27 \%-42 \%$ in phase II, thus being among the most active drugs for this disease. Cisplatin is a good candidate for combination with paclitaxel due to non-overlapping toxicity profiles and mechanisms of action, and in view of the clinical activity of both agents. While paclitaxel mainly affects the mitotic process, cisplatin primarily acts as an alkylating agent, explaining additive cytotoxic effects and a lack of cross-resistance in some cell lines. ${ }^{3}$

The combination of paclitaxel and cisplatin was impressively active, with an intent-to-treat response rate of $53.7 \%$. the objective response rate was $71 \%$ (95\% CI: $52-86$ ), with complete response of $14 \%$ and partial response in $36 \%$. This was in comparison to the current study which showed $70.5 \%$ response rate with complete response in $17.6 \%$ and partial response in $52.9 \%{ }^{14}$. The slightly higher incidence of complete response seen in the current study may be due to use of the weekly paclitaxel.

Another feasible combination of docetaxel $\left(80 \mathrm{mg} / \mathrm{m}^{2}\right.$ day 1 ), cisplatin (40 mg/m² day 1 and 2) and infusional 5fluorouracil (1000 mg/m² day 1-3), achieved 12 responses in 16 mostly non-pretreated patients with squamous cell or nasopharyngeal carcinoma. Among 22 non-pretreated evaluable patients in ECSG trial, the overall response rate was $86.4 \%$ (95\% CI: $65 \%-97 \%)$. This subgroup of patients is most likely to achieve an objective response to treatment, as several induction chemotherapy trials have shown, resulting in clinical complete responses above $60 \%$ with total response rates exceeding $80 \%{ }^{13-17}$. This in contrast to another study done by Schrijvers et al. who reported response rate of $70.8 \%$ in the form of partial response only by two different levels of doses for the combination of docetaxel, cisplatin and 5-FU. ${ }^{16}$

In the current study, we decided to perform a separate analysis of response and survival data of patients with cancer of nasopharynx and nasal cavity (NPC group) and those with cancer of other sites of the head and neck region (Non-NPC) because NPC is considered a distinct entity with a unique biological behavior. This was confirmed in the current study by the multivariate analysis which revealed that the most important determinant of response to chemotherapy was the site of the tumour whether NPC or non-NPC. The overall response rate in non-NPC patients was $66.7 \%$, which appears to be higher than that reported in Fountzilas et al. ${ }^{5}$ using the two drugs alone (23\%) (but not weekly paclitaxel like the current study) and than that reported with the combination of carboplatin and fluorouracil (21\%). Added to that, response in both NPC and non-NPC where CR and PR were found to be $14 \%$ and $43 \%$ in NPC, respectively, versus $36.4 \%$ and 54.6 in the current study, and $6 \%$ and $17 \%$ in non-NPC group, respectively, versus $9.5 \%$ and $57 \%$ in the current study. The statistical higher percentage of response in the current study may be due to weekly regimen of paclitexl used instead of 4 weekly regimen and the integration of cisplatin instead of carboplatin used in the former study.

Up to our knowledge, a unique analysis was done in the current study using multivariate analysis and showed that in addition to the site of the tumour whether NPC or non-NPC, performance status, nodal status and age were found to be very deterministic in the response to chemotherapy given in the current study. This was partially prevealed in table (5) in such way the complete responders were $66.7 \%$ nasopharyngeal tumours, aged $43.7+5.8$ years old, with all performance status not more than $1,66.7 \%$ T3 but the most important that all had nodal status between N0-2 and $66.7 \% \mathrm{~N} 0-1$, in comparison to the non-responders were $87.5 \%$ non-NPC, aged $51+8.9$ years old (i.e. significant older), $25 \%$ of them had PS 2 , $50 \%$ had $\mathrm{T} 4$ but more important that N3 were found in $37.5 \%$ and $\mathrm{N} 2$ in $25 \%$ (i.e. $62.5 \%$ had N2-3).

Although concurrent chemoradiation has become the standard of care for advanced and/or unresectable head and neck carcinoma patients, the best drug and schedule of chemoradiation remains to be determined. This trial was designed to test the efficacy and toxicity of a regimen of weekly paclitaxel and cisplatin concurrent with radiation in a group of patients with advanced HNSCC. Most of the patients were stage III and IV and considered unresectable by the referring surgeon together with $83.4 \%$ of them were node positive. Despite these 
unfavorable patient characteristics, this regimen showed an encouraging tumor response rate and acceptable survival results.

In addition, Chemoradiation in the current study, increases responders from $70.5 \%$ to $96.7 \%$ shift of nonresponders to chemotherapy to responders in such way that complete response was increased significantly from $17.6 \%$ to $56.7 \%$ and partial response decreased from $52.9 \%$ to $40 \%$ (due to increase in CR).

Hennequin and Favaudon recently reviewed the biological mechanisms of interaction between chemotherapy and radiotherapy ${ }^{11}$. These include interactions at the molecular, cellular, and tissue levels. At the molecular level, radiation and drugs cooperate to target DNA, by increasing DNA damage and interfering with DNA repair. At the cellular level, chemoradiation may induce cytokinetic cooperation. Radiosensitivity changes during the phases of the cell cycle. The $\mathrm{S}$ phase is the most radioresistant, whereas S-phase cells are highly sensitive to several anticancer drugs. This is the reason why a greater cell kill is observed when proliferating cells are exposed to drugs and radiation in close temporal proximity. ${ }^{11}$

There is one additional mechanism of action that may be ascribed to ACR. Split-course radiotherapy is considered suboptimal because of the tumor repopulation that occurs during treatment breaks, which negatively affects treatment results ${ }^{16,17}$. However, in ACR, the breaks between radiotherapy treatments are filled up with chemotherapy, the activity of which is enhanced in rapidly proliferating tissues, such as repopulating tumors. Therefore, a cytokinetic mechanism of cooperation, exploiting tumor repopulation, may be at work in ACR. At the tissue level, cooperation between radiation and the chemotherapy drugs is the result of rapid tumor shrinkage and reoxygenation resulting from an improved blood supply. This effect could be related to a reduction in interstitial pressure. Interstitial pressure usually increases in tumor tissues and leads to vascular collapse $^{11}$, thus compromising the blood supply, which is already defective because of poorly functioning vasculature (immature structure of the vessels as a result of imperfect neoangiogenesis). In the case of rapid tumor mass reduction, interstitial pressure may be reduced and blood flow improved.

The issue of neoadjuvant chemotherapy is very controversial, since the majority of studies failed to improve survival. However as trial to compare impact of different combination on survival, the current study showed that median survival was not yet achieved due to short period of follow up (24 months), however, survival at 24 months was found to be $83 \%$, 91\%, and $79 \%$ for all patients, NPC and non NPC respectively in comparison to $40.9 \%$ reported by Schrijvers et $\mathrm{al}^{12}$ using combination of docetaxel, cisplatin and 5-FU. On the other hand, Fountzilas et al. ${ }^{5}$ reported that at the time of the analysis, median survival had not been reached in NPC while it was 7.3 months in non-NPC patients using 3hours infusion of paclitaxel together with cisplatin. On the other hand, Meriano et al. ${ }^{2}$ reported 2- and 3-year actuarial overall survival to be $75.5 \%$ and $61.4 \%$ and $65 \%$ in Benasso et al. ${ }^{18}$ [using combination of alternating gemcitabine and cisplatin with gemcitabine and radiation in stage IV squamous cell carcinoma of the head and neck] which is very similar to current study. The reasons for difference is the use of both weekly dose of paclitaxel together with the application of radiotherapy which showed higher incidence of complete response. In addition, the intentionto-treat $\mathrm{CR}$ rate $(17.6 \%)$, the actuarial local control ( $76.9 \%$ at 2 years) and OS achieved ( $83 \%$ at 2 years) are remarkable if we consider that patients enrolled in the present trial had poor-prognosis head and neck cancer.

In the current study, combined chemo-radiation was used to minimize toxicities. The main side effects were gastrointestinal toxicity, stomatitis and haematological toxicity. However in comparison to Merlano et al. ${ }^{2}$ who used combination of paclitaxel, cisplatin and 5-FU together with radiotherapy, hematological toxicity was more or less the same with less severity of leuopenia in the current study. On the other hand, severity of gastrointestinal toxicity was found to be higher in the current study. Similar dermatoligcal toxicity in the form of alopecia, sensory neuropathy, and hypersensitivity and also renal function affection. The skin toxicities and stomatitis were the main cause of interruption of paclitaxel during the management.

\section{CONCLUSION}

There is a renewed interest in neo-adjuvant chemotherapy, in particular when it is followed by concurrent chemoradiation programs. This will undoubtedly lead to more toxicity, and methods to reduce or overcome these toxicities should be further explored. Induction chemotherapy with weekly paclitaxel and threeweekly cisplatin followed by concomitant chemoradiation proved its efficacy in short and intermediate term follow up in patients with unresectable head and neck tumours. Primary site, nodal status, performance status together with age are very deterministic for response of induction protocol.

\section{REFERENCES}

1. Merlano M. Alternating chemotherapy and radiotherapy in locally advanced head and neck cancer: An alternative? Oncologist.2006;11(2):146-51.

2. Merlano M, Russi EG, Numico G, Colantonio I, Garrone O, Pelissero A, et al. Paclitaxel, cisplatin, 5-fluorouracil and radiotherapy in the management of advanced squamous cell 
carcinoma of the head and neck: A phase II trial. Radiother. Oncol.2005;75(2):193-6.

3. Forastiere A, Koch W, Trotti A, Sidransky D. Head and neck cancer. N.Engl.J.Med.2001;345(26):1890-900.

4. Schoffski P, Catimel G, Planting AS, Droz JP, Verweij J, Schrijvers $\mathrm{D}$, et al. Docetaxel and cisplatin: An active regimen in patients with locally advanced, recurrent or metastatic squamous cell carcinoma of the head and neck. Results of a phase II study of the EORTC Early Clinical Studies Group. Ann.Oncol.1999;10(1):119-22.

5. Fountzilas G, Skarlos D, Athanassiades A, Kalogera Fountzila A, Samantas E, Bacoyiannis C, et al. Paclitaxel by three-hour infusion and carboplatin in advanced carcinoma of nasopharynx and other sites of the head and neck. A phase II study conducted by the Hellenic Cooperative Oncology Group. Ann. Oncol.1997;8(5):451-5.

6. Benasso M, Corvo R, Ponzanelli A, Sanguineti G, Ricci I, Pallestrini E, et al. Alternating gemcitabine and cisplatin with gemcitabine and radiation in stage IV squamous cell carcinoma of the head and neck. Ann.Oncol.2004;15(4):646-52.

7. Hitt R, Paz Ares L, Brandariz A, Castellano D, Pena C, Millan $\mathrm{JM}$, et al. Induction chemotherapy with paclitaxel, cisplatin and 5-fluorouracil for squamous cell carcinoma of the head and neck: Long-term results of a phase II trial. Ann.Oncol.2002;13(10):166573.

8. Al Sarraf M, LeBlanc M, Giri PG, Fu KK, Cooper J, Vuong T, et al. Chemoradiotherapy versus radiotherapy in patients with advanced nasopharyngeal cancer: Phase III randomized Intergroup study 0099. J.Clin.Oncol.1998;16(4):1310-7.

9. Monnerat C, Faivre S, Temam S, Bourhis J, Raymond E. End points for new agents in induction chemotherapy for locally advanced head and neck cancers. Ann.Oncol.2002;13(7):9951006.
10. Jeremic B, Shibamoto Y, Milicic B, Nikolic N, Dagovic A, Aleksandrovic J, et al. Hyperfractionated radiation therapy with or without concurrent low-dose daily cisplatin in locally advanced squamous cell carcinoma of the head and neck: A prospective randomized trial. J.Clin.Oncol.2000;18(7):1458-64.

11. Hennequin C, Favaudon V. Biological basis for chemoradiotherapy interactions. Eur.J.Cancer.2002;38(2):223-30.

12. Schrijvers D, Van Herpen C, Kerger J. Phase I-II study with docetaxel (D), cisplatin (C) and 5-fluorouracil (5-FU) in patients with locally advanced inoperable squamous cell carcinoma of the head and neck (SCCHN). J.Clin.Oncol.1999;18(394a):1524.

13. Polee MB, Tilanus HW, Eskens FA, Hoekstra R, Van der Burg ME, Siersema PD, et al. Phase II study of neo-adjuvant chemotherapy with paclitaxel and cisplatin given every 2 weeks for patients with a resectable squamous cell carcinoma of the esophagus. Ann. Oncol.2003;14(8):1253-7.

14. Posner MR. Paradigm shift in the treatment of head and neck cancer: the role of neoadjuvant chemotherapy. Oncologist.2005;10 (Suppl 3):11-9.

15. Kaplan EL, Meier P. Nonparametric estimation from incomplete observations. J.Am.Stat.Assoc.1958;53(282):457-81.

16. Argiris A, Smith SM, Stenson K, Mittal BB, Pelzer HJ, Kies MS, et al. Concurrent chemoradiotherapy for N2 or N3 squamous cell carcinoma of the head and neck from an occult primary. Ann. Oncol.2003;14(8):1306-11.

17. Vermorken JB. Medical treatment in head and neck cancer. Ann. Oncol.2005;16 (Suppl 2):ii258-64.

18. Benasso M, Ponzanelli A, Merlano M, Numico G, Ricci I, Vigo V, et al. Paclitaxel, cisplatin and 5-fluorouracil in recurrent squamous cell carcinoma of the head and neck: A phase II trial from an Italian cooperative group. Acta Oncol.2006;45(2):168-74. 